\title{
Small Intestinal Bacterial Overgrowth following Subtotal Colectomy for Colon Cancer
}

\author{
Jigar Bhagat W and Satish Rao SC*
}

Division of Gastroenterology and Hepatology, Department of Internal Medicine, Augusta University, Augusta, Georgia, USA

${ }^{*}$ Corresponding author: Satish Rao SC, Division of Gastroenterology and Hepatology, Department of Internal Medicine, Augusta University, Digestive Health Center, 1481 Laney-Walker Blvd, Augusta, GA 30912, USA, Tel: 7067212238; Fax: 7067210331; E-mail: srao@augusta.edu

Received: July 31, 2017; Accepted: August 09, 2017; Published: August 16, 2017

Citation: Jigar Bhagat W, Satish Rao SC (2017) Small Intestinal Bacterial Overgrowth Following Subtotal Colectomy for Colon Cancer. Colorec Cancer. Vol.3 No.2:13.

Copyright: (C) 2017 Jigar Bhagat W, et al. This is an open-access article distributed under the terms of the Creative Commons Attribution License, which permits unrestricted use, distribution, and reproduction in any medium, provided the original author and source are credited.

\section{Abstract}

Background: Increasingly colon cancers are diagnosed early and treated surgically with excellent survival. However, after surgery, one third of patients report chronic unexplained gastrointestinal symptoms and poor quality of life.

Aim: To determine if small intestinal bacterial overgrowth could explain these symptoms is unclear.

Methods: We present a series of patients who developed chronic daily abdominal pain, gas, bloating and diarrhea after subtotal colectomy for colorectal cancer. Evaluations with CAT scan, endoscopies and empirical treatments were ineffective. We performed either a glucose breath test by administering $75 \mathrm{~g}$ glucose in $250 \mathrm{~mL}$ of water and collecting breath samples every 15 min for 2 hours and/or upper endoscopy with duodenal aspiration and culture for aerobic, anaerobic and fungal organisms. We found that all of these patients had small intestinal bacterial overgrowth. A two-week course of antibiotics led to complete resolution of symptoms.

Conclusion: Our cases illustrate that small intestinal bacterial overgrowth is a common yet poorly recognized problem in patients following subtotal colectomy. This condition can be readily identified with breath test and/or duodenal aspirates and culture. Prompt diagnosis and treatment of this condition can be very effective in ameliorating symptoms.

\section{Background}

Colon cancer is the second most common cancer in USA leading to 50,000 deaths annually. With increasing awareness and screening, majority of the colon cancers are diagnosed at early stage with $\sim 90 \% 5$-year survival after subtotal colectomy (https://seer.cancer.gov/statfacts/html/colorect.html). In addition to the rare but expected post-surgical complications such as infection, sepsis, bleeding, prolonged ileus and anastomotic obstruction or leaks, some patients develop chronic unexplained gastrointestinal symptoms. In a few retrospective studies, patients who underwent colectomy for malignant and non-malignant etiologies reported chronic abdominal pain, bloating, excess gas and diarrhea [1,2] with significant negative impact on their quality of life [3]. However, there is limited data regarding the long-term follow up of these patients with regards to their bowel symptoms and quality of life. Management of such symptoms could be challenging, costly and exposes cancer survivors to unnecessary diagnostic tests.

One possible explanation for the persistent unexplained GI symptoms could be the development of small intestinal bacterial overgrowth (SIBO), due to a loss of the ileocecal valve barrier and/or intestinal dysmotility or adhesions. SIBO is characterized by the presence of abdominal pain, gas, bloating and diarrhea together with abnormally elevated bacterial counts in the small bowel $[4,5]$. The premature fermentation of dietary carbohydrates and the deconjugation of bile salts by the bacteria in the small bowel, leads to gas, bloating and osmotic diarrhea.

Here, we report four patients who underwent subtotal colectomy for colorectal cancer and presented with unexplained gastrointestinal symptoms comprising of abdominal pain, gas, bloating, belching and diarrhea.

\section{Methods}

Over one year, we identified patients who were referred to our motility center with unexplained GI symptoms and who had undergone subtotal colectomy for colorectal cancer or for suspected malignant colon mass. This analysis was approved by our institutional review board, No 659642-3.

The patient's clinical symptoms and diagnostic work-up is detailed under each case. Briefly all subjects were ambulatory and had normal upper endoscopy, colonoscopy with intact surgical anastomosis, abdominal computerized automated tomography (CAT) scan and/or ultrasound exam. Patients also tested negative for celiac disease, hypo or hyperthyroidism 
and neuroendocrine disease. None of the patient reported narcotic, probiotic or antibiotic use within the 6 weeks prior to the testing. All patients completed a validated bowel symptom questionnaire [6] assessing abdominal pain, belching, bloating, fullness, indigestion, nausea, diarrhea, vomiting and gas for its frequency, intensity and duration on a 0-3 scale for each with maximum total score of 9. Testing for SIBO consisted of performing either the diagnosed using glucose (fructose for diabetic patient) breath test and/or distal duodenal aspirates (DDA) and bacterial cultures using standardized methods described previously [7-9]. In brief, $75 \mathrm{~g}$ of glucose dissolved in $250 \mathrm{~mL}$ of water was given to subjects. Breath samples were collected at baseline and at 15 min interval for total of 2 hours. The subjects reported symptoms during the test. For DDA, during upper endoscopy a $2 \mathrm{~mm}$ Liguory catheter was passed through the scope to the most distal portion of the duodenum under sterile environment and $3 \mathrm{ml}$ of small bowel fluid was aspirated and sent for aerobic, anaerobic and fungal cultures. During a breath test, a rise in $>20 \mathrm{ppm}$ of hydrogen or $>10 \mathrm{ppm}$ of methane was considered positive for SIBO [8]. From DDA, a colony count of $\geq 10^{3} \mathrm{CFU} / \mathrm{mL}$ was considered positive for SIBO. Based on a diagnosis of SIBO with either method, patients were then treated with appropriate antibiotic(s) for 14 days and followed up with a clinic visit or a phone call after 6 weeks (Table 1).

\section{Cases}

Table 1 Individual cases are summarized.

\begin{tabular}{|c|c|c|c|c|c|c|c|}
\hline Case & Age & Gender & $\begin{array}{l}\text { Site of } \\
\text { colectomy for } \\
\text { colon cancer }\end{array}$ & Symptom profile & SIBO & $\begin{array}{l}\text { SIBO with } \\
\text { breath test }\end{array}$ & $\begin{array}{l}\text { SIBO with } \\
\text { Duodenal } \\
\text { aspiration }\end{array}$ \\
\hline 1 & 75 & $\mathrm{~F}$ & Right & $\begin{array}{l}\text { Abdominal pain, bloating, fullness, } \\
\text { nausea, belching, indigestion, } \\
\text { diarrhea, gas and vomiting }\end{array}$ & YES & YES & Not performed \\
\hline 2 & 77 & M & Right & Gas & YES & YES & Not performed \\
\hline 3 & 47 & $F$ & Left & $\begin{array}{l}\text { abdominal pain, cramping, bloating, } \\
\text { fullness, nausea, belching, } \\
\text { indigestion, diarrhea, and gas }\end{array}$ & YES & Not performed & YES \\
\hline 4 & 85 & M & Left & $\begin{array}{l}\text { Abdominal pain, cramping, bloating, } \\
\text { fullness, nausea, belching, } \\
\text { indigestion, diarrhea, and gas }\end{array}$ & YES & YES & YES \\
\hline
\end{tabular}

\section{Case 1}

A 75-year-old female with a history of right hemicolectomy and ileo-colonic anastomosis for a colonic mass, and partial left sided colectomy with primary anastomosis for colonic obstruction several years ago and cholecystectomy presented with complaints of abdominal bloating, gas and diarrhea for over one year. She had normal CT scan, upper endoscopy and colonoscopy prior to referral, other than post-surgical changes. She also tested negative for pancreatic insufficiency, malabsorption syndrome and routine stool cultures. Her symptoms prior to testing and their severity (0-9) were: Abdominal pain (5), bloating (3), fullness (3), nausea (3), belching (3), indigestion (6), diarrhea (9), gas (9) and vomiting (3). She underwent glucose breath test which showed a significant rise in hydrogen gas at $45 \mathrm{~min}$ with associated worsening symptoms indicating SIBO, and reproduction of her home symptoms (Figure 1).

\section{Case 2}

A 77-year-old male with hepatic flexure adenocarcinoma underwent right hemicolectomy and ileocolic anastomosis. Several years later he presented with long standing complaints of daily gas and constipation. He had persistent gas after effective treatment of constipation. He was tried on simethicone with no improvement. His CT scan, upper endoscopy and colonoscopy were unremarkable apart from post-op changes. He underwent glucose breath test showing early rise of methane gas from $12 \mathrm{ppm}$ at baseline to $25 \mathrm{ppm}$ at 30 min indicating proximal SIBO.

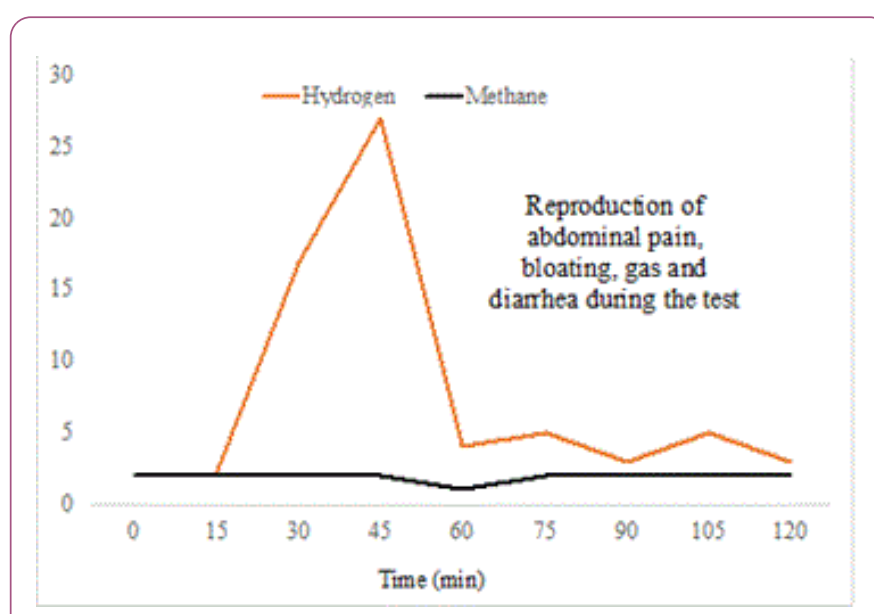

Figure 1: Figure shows the glucose breath test profile from case 1 . After ingestion of glucose there is a significant rise in breath hydrogen values starting at 30 minutes with no change in breath methane levels, together with reproduction of patient's baseline symptoms.

\section{Case 3}

A 47-year-old female with history of sigmoid cancer $s / p$ partial colectomy at the age of 40 presented with 7 years of 
unexplained daily abdominal pain, bloating, gas and diarrhea with 3-4 non-bloody bowel movements with Bristol stool class 4. She had abdominal CT scan, upper endoscopy and colonoscopy performed without identifiable diagnosis. She was placed on daily proton pump inhibitor (PPI) without improvement in her symptoms. Prior to testing for SIBO, her symptoms with total severity score were: abdominal pain (8), cramping (8), bloating (9), fullness (7), nausea (4), belching (8), indigestion (3), diarrhea (3), and gas (9). Patient underwent glucose breath test which was negative. Due to high level of suspicion, she underwent upper endoscopy with DDA and culture. She was noted to have polymicrobial overgrowth of $\geq$ 103 colony forming unit (CFU) growth of Streptococcus viridans, Haemophilus parainfluenza, non-gonococcal Neisseria species, unspecified anaerobic gram-negative bacilli and $105 \mathrm{CFU}$ growth of unspecified anaerobic gram-negative cocci per $\mathrm{mL}$ of DDA.

\section{Case 4}

An 85-year-old male with left colon cancer and left hemicolectomy 12 years previously presented with 10-year history of daily abdominal pain, bloating, abdominal distention, nausea, and vomiting. He was thought to have intermittent small bowel obstructions due to internal hernia and post-surgical adhesions. He underwent laparoscopic explorations and removal of adhesions and cholecystectomy without improvement in his symptoms. Due to his age and surgical risk, he was referred to neurogastroenterology. Patient reported several severe symptoms: Abdominal pain (5), cramping (8), bloating (9), fullness (9), nausea (9), belching (8), indigestion (9), diarrhea (5), and gas (9). He underwent glucose breath test and upper endoscopy with DDA. The hydrogen breath test was positive with a marked rise in hydrogen levels in breath samples collected at 45 minutes. Patient's upper endoscopy was unremarkable. The DDA was cultured which showed 105 CFU growth of Escherichia coli, Streptococcus species alpha-hemolytic and Streptococcus viridans.

\section{Discussion}

Although colectomy with ileo-colonic anastomosis is often life saving for colon cancer, up to a third of patients may experience unexplained gastrointestinal symptoms. Here, we describe four patients all of whom underwent colectomy for colon cancer. After surgery, and many years later all of these patients developed new symptoms that were often described as IBS or as a functional gastrointestinal disorder by their primary care physician or surgeon or gastroenterologist. Routine tests including endoscopy and CAT scans were all negative. These patients suffered significant daily symptoms without diagnosis as illustrated by subject 1 , and failed empirical trials of treatments and was eventually referred to a motility center.

Our testing with glucose breath test and/or duodenal aspiration and culture identified the presence of SIBO in all 4 subjects. Furthermore, there was reproduction of symptoms during the breath test in some of the subjects indicating correlation of symptoms with SIBO. These cases illustrate that SIBO could be a common problem in patients following colectomy, especially after ileo-colonic anastomosis. There are two plausible reasons. One, the ileo-cecal valve serves as an important barrier between the ileum and cecum that not only regulates flow of contents from the small bowel to the cecum but also prevents reflux of contents and transmigration of colonic contents and bacteria into the small bowel. Loss of ileocecal valve is a known risk factor for the development of SIBO $[10,11]$. And second is that subtotal colectomy alters the natural anatomy together with postoperative changes such as adhesions may lead to altered gut motility resulting in increased risk of SIBO.

Thus, a loss of the critical anatomical barrier following colectomy or intestinal dysmotility or both may predispose patients with colectomy to recurrent small intestinal bacterial overgrowth. Our cases illustrate that this problem can be identified in most cases with either the non-invasive glucose breath tests or duodenal aspiration and culture. A positive diagnosis not only establishes definitive diagnosis but can guide management especially with aspiration, culture and antibiotic sensitivity.

Our cases underscore the finding that although colectomy is a useful and safe procedure, the altered anatomy stemming either from the ileo-colonic or colo-colonic anastomosis inadvertently predisposes these patients to unforeseen consequences such as SIBO or perhaps small intestinal fungal overgrowth (SIFO). The key is awareness and recognition of this problem followed by appropriate treatment can lead to significant amelioration of symptoms. Some patients however will require recurrent or long-term antibiotic therapy.

\section{References}

1. Petrone $P$, Sarkisyan G, Fernández $M$, Coloma E, Akopian G, et al. (2011) Small intestinal bacterial overgrowth in patients with lower gastrointestinal symptoms and a history of previous abdominal surgery. Arch Surg 146: 444-447.

2. Theodoropoulos GE, Papanikolaou IG, Karantanos T, Zografos G (2013) Post-colectomy assessment of gastrointestinal function: a prospective study on colorectal cancer patients. Tech Coloproctol 17: 525-536.

3. FitzHarris GP, Garcia-Aguilar J, Parker SC, Bullard KM, Madoff RD, et al. (2003) Quality of life after subtotal colectomy for slowtransit constipation: both quality and quantity count. Dis Colon Rectum 46: 433-440.

4. Sachdev AH, Pimentel M (2013) Gastrointestinal bacterial overgrowth: pathogenesis and clinical significance. Ther Adv Chronic Dis 4: 223-231.

5. Dukowicz AC, Lacy BE, Levine GM (2007) Small intestinal bacterial overgrowth: a comprehensive review. Gastroenterol Hepatol 3: 112-122.

6. Choi YK, Kraft N, Zimmerman B, Jackson M, Rao SS (2008) Fructose intolerance in IBS and utility of fructose-restricted diet. J Clin Gastroenterol 42: 233-238.

7. Rezaie A, Buresi M, Lembo A, Lin H, McCallum R, et al. (2017) Hydrogen and Methane-Based Breath Testing in Gastrointestinal 
Disorders: The North American Consensus. Am J Gastroenterol 112: 775-784.

8. Erdogan A, Rao SS, Gulley D, Jacobs C, Lee YY, et al. (2015) Small intestinal bacterial overgrowth: duodenal aspiration vs glucose breath test. Neurogastroenterol Motil 27: 481-489.

9. Rezaie A, Pimentel M, Rao SS (2016) How to Test and Treat Small Intestinal Bacterial Overgrowth: an Evidence-Based Approach. Curr Gastroenterol Rep 18: 8.
10. Jacobs C, Coss AE, Attaluri A, Valestin J, Rao SS (2013) Dysmotility and proton pump inhibitor use are independent risk factors for small intestinal bacterial and/or fungal overgrowth. Aliment Pharmacol Ther 37: 1103-1111.

11. Klaus J, Spaniol U, Adler G, Mason RA, Reinshagen M, et al. (2009) Small intestinal bacterial overgrowth mimicking acute flare as a pitfall in patients with Crohn's Disease. BMC Gastroenterol 9: 61. 